\title{
Comments on "An Ontological Evaluation of the OML Metamodel" by A.L. Opdahl, B. Henderson-Sellers and F. Barbier
}

\author{
Arne Sølvberg \\ Trondheim, Dept.Computer Science \\ The Norwegian University of Science and Technology (NTNU) \\ N-7491 Trondheim, Norway
}

The starting point of the paper is to analyse the constructs of the OPEN Modeling Language (OML) which is developed with one of the co-authors as the driving force. The analysis is done in terms of the FRISCO ontology (which is the subject of the ISCO conference series) and of the BungeWand-Weber (BWW) ontology. The result of the analysis is summed up in four tables and is a comprehensive description of OML.

The discussion in the paper is not only about OML, but is also a discussion and comparison of the conceptual contents of FRISCO and $\mathrm{BWW}$. For the latter discussion OML is used as a vehicle for discussing the relative merits of the two proposals for information systems ontologies. Both discussions (the OML clarification, and FRISCO vs. BWW) are interesting and useful. The latter, however, appears more as an afterthought than as having been planned for when the reported investigation started. For this reader the FRISCO vs. BWW discussion is the more interesting of the two, and would therefore like to see a continuation of the work beyond what is currently reported. The following remarks are to this end.

The OML analysis is verbal. The various OML constructs are compared to their FRISCO and BWW counterparts not through formal expressions but with words. FRISCO invites to a formal comparison through the formal 
definitions of its ontological constituents. A formal comparison between OML and FRISCO should be within reach. This is a difficult job, however, and is without precedent as far as I am aware of. It should nevertheless be done in order to remove the vagueness (implied by necessity) of a verbal comparison. The same goes for the FRISCO vs. BWW comparison. We have proceeded so far now in our deliberations on concept modeling that increased formality appears to belong to the next step in IS research.

There is disturbing vagueness in parts of the basis of BWW and FRISCO which should be straightened out. E.g., it is stated in the paper that BWWthings are "concrete" and FRISCO-things are "conceptions". What this really means is difficult to understand. The paper does also not offer much help in this respect. So we have to live with a corresponding vagueness in the analysis of OML, e.g., an OML-object is a BWW-thing, but may also be an FRISCO-instance, which is a conception and not a concrete thing (table 1, first entry).

There is no reason to give the authors full responsibility for the vagueness. It is unavoidable because of the inherent vagueness in either FRISCO, BWW or in both of the frameworks. Further research should remove such discrepancies. The authors of the current paper have shown that they may be able to contribute to this end. They are hereby encouraged to do exactly that in their future research. 